\title{
Die Patella in der Knieendoprothetik
}

ie Patella spielt in der Knieendoprothetik bekanntlich eine bedeutende Rolle und nahezu jedes Jahr wird dieses Thema auf Kongressen oder Symposien behandelt. In med-line gelisteten Journals wurden zwischen 1993 und 1997 knapp 100 Arbeiten publiziert, die sich ausschließlich mit dem Thema Patellarückflächenersatz in der Knieendoprothetik kritisch auseinandersetzen.

Mit unserer Auswahl und Zusammenstellung der Beiträge soll dem Leser geholfen werden, sich ein umfassendes Bild über den aktuellen Wissensstand zu verschaffen.

Die Ausführungen von Herrn Schroeder-Boersch zum aktuellen Stand der Kontroverse sollen dem Leser das Hintergrundwissen vermitteln, um ihm die Einordnung der nachfolgenden Beiträge zu erleichtern.

Anschließend folgt der Beitrag der Arbeitsgruppe um Komistek, Dennis und Mabe aus Denver, Colorado, die schon seit einigen Jahren versuchen, die Bewegungsabläufe des Kniegelenkes genauer darzustellen. Erstmals wird unter in-Vivo- und Vollbelastungsbedingungen das Verhalten von endoprothetisch ersetzten Kniegelenken untersucht. Bei dem in diesem Heft veröffentlichten Beitrag wurde auf eine bislang nicht hinreichend bekannte „Entkopplung" und
Abhebung der Patella vom femoralen Gleitlager bei voller Streckung berichtet. Möglicherweise trägt dieses Phänomen zu einem Teil der postoperativ beobachteten Probleme mit der Patella bei.

Ein weiteres, bisher noch nicht hinreichend erfaßtes Problem ist die veränderte propriozeptive Fähigkeit des Kniegelenkes bei zunehmender Arthrose. Zwar geht der Beitrag von Pap, Machner und Awiszus nicht explizit auf die veränderte Propriozeption nach Implantation einer Knieprothese ein, es fanden sich aber mehr Defizite der propriozeptiven Leistungen bei zunehmender Retropatellararthrose. Wegen der großen interindividuellen Schwankungen der Werte bietet eine präoperative Messung der propriozeptiven Fähigkeiten bislang noch keine Hilfe bei der Entscheidung pro oder contra Patellarückflächenersatz.

Scheller et al. zeigen noch einmal die wesentlichen Implantationsschritte auf, die für eine erfolgreiche Implantation mit bestmöglicher Position der $\mathrm{Pa}$ tella im "neuen" femoropatellaren Gleitlager erforderlich sind.

Anschließend folgen zwei Arbeiten von schweizerischen Kollegen, die jeweils erfolgreich auf einen Patellarückflächenersatz verzichtet haben. Beide Autoren beschreiben eine erhebliche Fähigkeit der nativ belassenen Patella sich remodellierend an das metallene femorale Gleitlager anzupassen. Gerber und Maenza konnten zudem die Bedeutung einer prä- und postoperativ bestehenden Lateralisierung der Patella auf das Auf- treten von postoperativen Beschwerden heraus arbeiten. Im Beitrag von Arnold et al. ist der von den Autoren standardmäßig verwendete laterale Zugang mit Ablösung der Tuberositas tibiae bemerkenswert. Ob das von dieser Arbeitsgruppe berichtete völlige Fehlen eines sonst in einem bestimmten Prozentsatz auftretenden anterioren Knieschmerzes mit diesem spezifischen Zugang zusammenhängt, muß dahingestellt bleiben.

Abgerundet wird die Thematik mit den Ergebnissen einer prospektiv randomisierten Arbeit aus unserer Klinik.

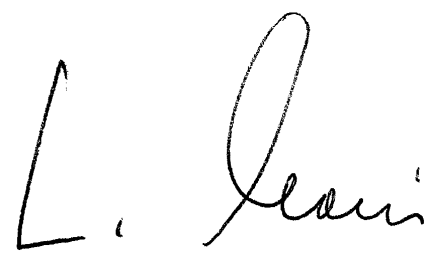

L.Jani

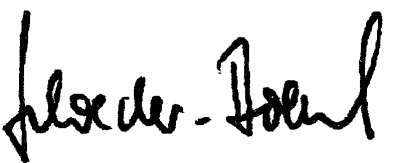

H. Schroeder-Boersch 